\title{
The Learning Curve for Biplane Medial Open Wedge High Tibial Osteotomy in 100 Consecutive Cases Assessed Using the Cumulative Summation Method
}

\author{
Do Kyung Lee, $\mathrm{MD}^{1}$, Kwang Kyoun Kim, $\mathrm{MD}^{1}$, Chang Uk Ham, $\mathrm{MD}^{1}$, Seok Tae Yun, $\mathrm{MD}^{2}$, \\ Byung Kag Kim, $\mathrm{MD}^{3}$, and Kwang Jun Oh, $\mathrm{MD}^{3}$ \\ ${ }^{1}$ Department of Orthopaedic Surgery, Konyang University Hospital, Konyang University College of Medicine, Daejeon; ${ }^{2}$ Department of Orthopaedic Surgery, Konkuk \\ University Medical Center, Konkuk University School of Medicine, Seoul; ${ }^{3}$ Department of Orthopaedic Surgery, Joint Center, SungMin Hospital, Incheon, Korea
}

Purpose: The purpose of this study was to investigate whether surgical experience could improve surgical competency in medial open wedge high tibial osteotomy (MOWHTO).

Materials and Methods: One hundred consecutive cases of MOWHTO were performed with preoperative planning using the Miniaci method. Surgical errors were defined as under- or overcorrection, excessive posterior slope change, or the presence of a lateral hinge fracture. Each of these treatment failures was separately evaluated using the cumulative summation test for learning curve (LC-CUSUM).

Results: The LC-CUSUM showed competency in prevention of undercorrection, excessive posterior slope change, and lateral hinge fracture after 27, 47, and 42 procedures, respectively. However, the LC-CUSUM did not signal achievement of competency in prevention of overcorrection after 100 procedures. Furthermore, the failure rate for overcorrection showed an increasing tendency as surgical experience increased.

Conclusions: Surgical experience may improve the surgeon's competency in prevention of undercorrection, excessive posterior slope change, and lateral hinge fracture. However, it may not help reduce the incidence of overcorrection even after performance of 100 cases of MOWHTO over a period of 6 years.

Keywords: Knee, Osteoarthritis, Osteotomy, Accuracy, Learning curve

\section{Introduction}

Surgical errors could be related with surgical outcomes, and accumulation of surgical experience in novice surgeons has been considered to reduce surgical errors. Achieving an appropriate level of competency with a surgical procedure is crucial for the patient's safety, providing high quality medical service, and pro-

Received August 13, 2017; Revised (1st) October 12, 2017;

(2nd) November 22, 2017; Accepted December 5, 2017

Correspondence to: Kwang Jun Oh, MD

Department of Orthopaedic Surgery, Joint Center, SungMin Hospital, 70

Sinsuk-ro, Seo-gu, Incheon 22789, Korea

Tel: +82-32-726-1276, Fax: +82-32-726-1109

E-mail: damioh@gmail.com

This is an Open Access article distributed under the terms of the Creative Commons Attribution Non-Commercial License (http://creativecommons.org/licenses/by-nc/4.0/) which permits unrestricted non-commercial use, distribution, and reproduction in any medium, provided the original work is properly cited. ducing satisfactory surgical outcomes ${ }^{1)}$. Recently, surgical competency has gained an increasing amount of attention ${ }^{1)}$.

Surgical competency of orthopedic surgeons has been commonly evaluated using clinical scores, operation times, errors in radiological parameters, and incidence of complications. However, this traditional method has demonstrated limited accuracy in evaluating a surgeon's competency. Recent studies have reported that the cumulative summation test for learning curve (LC-CUSUM) analysis could be used to evaluate the learning curve for orthopedic procedures ${ }^{2-4)}$. The LC-CUSUM analysis could determine whether a surgeon's performance has achieved a predefined level of competence; surgeons could also use it for self-monitoring and continuous quality improvement in surgical procedures $^{2,4,5)}$. Several authors ${ }^{3,4,6)}$ reported that the LC-CUSUM analysis in orthopedic surgery could be a useful method to evaluate learning curves and surgical competence; however, the method is uncommon in the field of orthopedics.

Medial open wedge high tibial osteotomy (MOWHTO) is a 
commonly performed surgical treatment for varus deformity and medial compartment knee osteoarthritis. Several factors such as achievement of planned correction and prevention of an excessive posterior slope change or lateral cortex hinge fracture have been considered to determine treatment success in biplane $\mathrm{MOWHTO}^{7-9)}$. Undercorrection is considered a surgical treatment failure because the weight bearing portion does not shift from the medial compartment to the lateral compartment; overcorrection could be a cosmetic problem and cause overloading of the lateral compartment. Both correction errors have been associated with unfavorable clinical results ${ }^{10)}$. The change in the posterior slope could affect knee biomechanics, resulting in increased strain on the anterior or posterior cruciate ligament, which, in turn, can lead to increased risk of both cruciate injuries ${ }^{9,11)}$. A lateral hinge fracture affects the stability at the osteotomy site, leading to loss of correction or an increased incidence of delayed union or nonunion of the osteotomy site $e^{12,13)}$.

The reported incidences of these surgical errors in MOWHTO vary widely ${ }^{7,8,14-19)}$. However, there has been no study evaluating the relationship between the incidence of these unfavorable surgical errors in MOWHTO and the level of competence of a surgeon. This study was designed to investigate whether the incidence of each surgical error could be reduced by improving competence of the surgeon using the LC-CUSUM analysis. The hypothesis was that the accumulation of surgical experience would decrease surgical errors in MOWHTO.

\section{Materials and Methods}

\section{Patients}

A single surgeon (KJO) performed biplane MOWHTO at our institution from February 2009 to November 2015. The surgical learning curve was evaluated retrospectively over the course of 100 consecutive procedures. All patients who underwent MOWHTO during the period were included in the study. The surgeon had previously performed 3 cases of MOWHTO since 2006. Because this volume was too small to reflect consecutive surgical experience, the first 3 cases was excluded from the study. On average, 16 cases of MOWHTO were performed per year.

The surgical indication was symptomatic Kellgren-Lawrence grade III or IV medial compartment knee osteoarthritis, without any evidence of progression into the lateral compartment.

\section{Preoperative Planning and Surgical Procedures}

The standard surgical technique was used in all cases. The amount of angular correction was decided preoperatively using a standing long bone X-ray according to the Miniaci method; the target correction angle was such that the weight bearing line (WBL) passes through $62.5 \%$ the width of the tibial plateau. Soft tissue laxity was not considered during preoperative planning. Varus or valgus stress X-rays were not evaluated preoperatively. An anteromedial vertical skin incision was performed at the center of the tibial shaft on the medial side. Before performing biplane osteotomy, a TomoFix plate (Synthes, Bettlach, Switzerland) was pre-matched with the tibial morphology, and the osteotomy level was decided using $\mathrm{C}$-arm guidance to ensure that the locking screws would not violate the articular surface or osteotomy site. The hamstrings were tagged and pulled downward to prevent injury and the superficial medial collateral ligament was partially released to expose the osteotomy site. Two guide pins were inserted from medial to lateral. Biplane osteotomy was performed along the guide pins, and coupled chisel technique was used. A sufficient osteotomy involving both the anterior and posterior cortices was performed to prevent lateral hinge fracture. The osteotomy site was extended as per preoperative planning, and coronal alignment was checked with a cable method. The targeted anterior opening gap was $67 \%$ of the posterior opening gap to prevent excessive posterior slope change. The osteotomy site was fixed with TomoFix plate and locking screws (Synthes).

\section{The Definition of Surgical Errors}

The definition of surgical errors was defined by three separate factors; correction error, excessive posterior slope change, and the presence of a lateral hinge fracture. Correction error consisted of under- and over correction., which were identified if the 6-month postoperative radiographs showed the WBL passed through a point less than $57.5 \%$ and more than $67.5 \%$ the width of the tibial plateau, respectively ${ }^{18)}$. Excessive posterior slope change was defined more than a $3^{\circ}$ change compared with the non-operated $\mathrm{knee}^{15)}$; a lateral hinge fracture was identified if a cortical breakage was seen on radiographs taken during the postoperative 5 days.

\section{Cumulative Summation Test Analysis}

The cumulative summation (CUSUM) test was applied to determine whether the surgeon achieved a predefined level of competence $^{6,20)}$. The test presumes that the surgeon is not proficient in the procedure at the beginning of monitoring and signals competency when the surgeon reaches an acceptable predefined level of performance. Therefore, the null hypothesis $\left(\mathrm{H}_{0}\right)$ was that the procedure was out of control (the performance was inadequate), and the alternative hypothesis $\left(\mathrm{H}_{1}\right)$ was that the procedure was in 
control (the performance was adequate).

Using a standard CUSUM curve, each case was plotted in sequence along the $\mathrm{X}$-axis. All measures were divided into success or failure based upon the predefined definitions. When success occurred, the variable ' $s$ ' was subtracted from the cumulative score. When failure occurred, the variable ' $1-s$ ' was added to cumulative score. Thus, an upward trend signified unacceptable performance, whereas a downward trend signified acceptable performance. The surgeon's performance was considered acceptable when the CUSUM score was located below the upper decision limit $(h 1)$. A score crossing $h 1$ suggested that the failure rate had reached an unacceptable level with a type I error probability of 0.05. A score crossing the lower decision limit $(h 0)$ suggested that the failure rate had reached an acceptable level with a type II error probability of 0.20 .

\section{LC-CUSUM Analysis}

The LC-CUSUM is a modified cumulative sum test designed to monitor the learning curve $e^{2,5)}$. Unlike a traditional CUSUM, the LC-CUSUM cannot cross the zero point, thus it remains at zero even when the surgeon continues to accumulate failures due to inadequate performance. The LC-CUSUM shows a downward trend as the surgeon's performance becomes adequate. The surgeon is considered to have achieved competence for the procedure when the LC-CUSUM reaches its ho.

Variables were divided into preset variables and calculated variables $(\text { Table } 1)^{6}$. Preset variables were decided based upon previous studies and included acceptable failure rate $(p 0)$, un- acceptable failure rate $(p 1)$, probability of type I error $(\alpha)$, and probability of type II error $(\beta)$. Calculated variables were decided based upon preset variables as per formula ${ }^{6}$ and included $h 0, h 1$, and variable ' $s$ ' (subtracted value from the cumulative score).

\section{Statistical Analysis}

Two separate surgeons (STY and BKK) measured the radiological parameters and decided whether each measurement was deemed a treatment failure or success based upon predetermined definitions. Interclass correlation coefficients (ICCs) for success or failure were evaluated to rule out observational bias. Consecutive cases were divided into two according the period: the first 50 cases were classified as early period and the second 50 as late period. The Student $t$-test was used to compare patient demographics between two periods; the frequency of treatment failure in each period was compared using the Fisher exact test. All statistical analyses were performed with IBM SPSS ver. 20.0 (IBM Corp,. Armonk, NY, USA). A p $<0.05$ was considered statistically significant. The ICC values were divided into good, fair and poor ( $>0.75$, good; $0.4-0.75$, fair; $<0.4$, poor). The interobserver reliability for each measurement was satisfactory and all measurements showed good correlation. The interobserver reliability of each radiologic measurement was as follows: overcorrection, 0.876; undercorrection, 0.880; excessive posterior slope change, 0.950; and lateral hinge fracture, 0.968 .

Each factor was separately evaluated using the LC-CUSUM. A standard CUSUM test was applied to ensure retention of proficiency if the surgeon reached competency as determined by the

Table 1. Cumulative Summation Equations and Variables

\begin{tabular}{|c|c|c|c|c|}
\hline Variable & Undercorrection & Overcorrection & Posterior slope & Lateral hinge fracture \\
\hline \multicolumn{5}{|l|}{ Preset } \\
\hline$p 0$-acceptable failure rate & 0.10 & 0.08 & 0.10 & 0.20 \\
\hline$p 1$-unacceptable failure rate & 0.20 & 0.18 & 0.20 & 0.30 \\
\hline a-probability of the type I error & 0.05 & 0.05 & 0.05 & 0.05 \\
\hline$\beta$-probability of the type II error & 0.20 & 0.20 & 0.20 & 0.20 \\
\hline \multicolumn{5}{|l|}{ Calculated } \\
\hline $\mathrm{P}=\ln (p 1 / p 0)$ & 0.69 & 0.81 & 0.69 & 0.4 \\
\hline $\mathrm{Q}=\ln [(1-p 0) /(1-p 1)]$ & 0.12 & 0.12 & 0.12 & 0.13 \\
\hline$s=\mathrm{Q} /(\mathrm{P}+\mathrm{Q})$ & 0.15 & 0.13 & 0.15 & 0.24 \\
\hline $1-s$ & 0.85 & 0.87 & 0.85 & 0.76 \\
\hline$a=\ln [(1-\beta) / \alpha]$ & 2.77 & 2.77 & 2.77 & 2.77 \\
\hline$b=\ln [(1-\alpha) / \beta]$ & 1.56 & 1.56 & 1.56 & 1.56 \\
\hline$h 0=-b /(\mathrm{P}+\mathrm{Q})$ & -1.93 & -1.68 & -1.93 & -2.94 \\
\hline$h 1=a /(\mathrm{P}+\mathrm{Q})$ & 3.42 & 2.98 & 3.42 & 5.22 \\
\hline
\end{tabular}


Table 2. Preoperative Patient Demographics

\begin{tabular}{|c|c|c|c|c|}
\hline Variable & Overall $(n=100)$ & Early period $(\mathrm{n}=50)$ & Late period $(\mathrm{n}=50)$ & p-value ${ }^{\mathrm{a})}$ \\
\hline Age (yr) & $59.6(5.9)$ & $59.4(6.0)$ & $59.8(5.9)$ & 0.688 \\
\hline Body mass index $\left(\mathrm{kg} / \mathrm{m}^{2}\right)$ & $26.9(3.1)$ & $26.6(2.8)$ & $27.2(3.3)$ & 0.330 \\
\hline Gender (female/male) & $87 / 13$ & $41 / 9$ & $46 / 4$ & 0.137 \\
\hline Preop posterior tibial slope $\left(^{\circ}\right)$ & $9.2(2.9)$ & $9.6(2.2)$ & $8.8(3.4)$ & 0.164 \\
\hline Preop mechanical HKA angle $\left(^{\circ}\right)$ & $5.1(3.0)$ & $4.8(2.9)$ & $5.5(3.1)$ & 0.205 \\
\hline Correction angle $\left(^{\circ}\right)$ & $9.8(3.6)$ & $9.3(3.3)$ & $10.3(3.9)$ & 0.149 \\
\hline
\end{tabular}

Values are presented as mean (standard deviation).

Preop: preoperative, HKA: hip-knee-ankle.

${ }^{a}$ Student $t$-test was performed for the comparison of patient demographics between the periods.

LC-CUSUM test. Based upon previous studies ${ }^{7,8,14-19,21)}, p 0, p 1$, variable ' $s$ ', $h 0$, and $h 1$ were calculated using the standard CUSUM formula ${ }^{6}$ (Table 1).

This study was approved by our Institutional Ethics Committee.

\section{Results}

\section{Comparison of the Failure Rate between Early and Late Periods}

There was no significant difference in preoperative patient demographics between the early and late periods (Table 2). There was no significant difference in undercorrection or overcorrection between the two periods $(\mathrm{p}<0.599$ and $\mathrm{p}<0.626$, respectively). There was also no significant difference in the rate of excessive posterior slope change and lateral hinge fracture between the two periods ( $\mathrm{p}<0.118$ and $\mathrm{p}<0.300$, respectively) (Table 3 ).

\section{The Cumulative Number of Failures}

The cumulative number of failures associated with undercorrection, excessive posterior slope change, and lateral hinge fracture demonstrated a decreasing tendency at the end of the late period. However, the cumulative number of failures associated with overcorrection demonstrated an increasing tendency at the end of late period (Fig. 1A and B).

\section{The LC-CUSUM and CUSUM}

The LC-CUSUM reached $h 0$ and signaled competency after 27 cases for undercorrection, indicating that adequate proficiency in avoiding undercorrection had been achieved. Consecutive standard CUSUM did not reach $h 1$, indicating that surgical proficiency was retained (Fig. 2A). However, The LC-CUSUM did not reach $h 0$ for overcorrection, therefore, the surgeon was not able to achieve adequate competence in preventing overcorrection (Fig. 2B).
Table 3. Comparison of the Failure Rate between Early and Late Periods

\begin{tabular}{lcrc}
\hline \multicolumn{1}{c}{ Variable } & Early period & Late period & p-value $^{\mathrm{a})}$ \\
\hline Undercorrection & $7 / 50(14)$ & $6 / 50(12)$ & 0.599 \\
Overcorrection & $15 / 50(30)$ & $20 / 50(40)$ & 0.626 \\
Posterior slope & $12 / 50(24)$ & $6 / 50(12)$ & 0.118 \\
Lateral hinge fracture & $14 / 50(28)$ & $7 / 50(14)$ & 0.300 \\
\hline
\end{tabular}

Values are presented as number (\%).

${ }^{a)}$ Fisher exact test was performed to compare the failure rate between the two periods.

The LC-CUSUM test also reached $h 0$ and signaled competency in avoiding an excessive posterior slope change and lateral hinge fracture after 47 and 42 cases, respectively. Consecutive CUSUM did not reach $h 1$ for any of these factors, indicating that proficiency was retained (Figs. 3 and 4).

\section{Discussion}

The principal findings of this study is that the surgeon was able to achieve and maintain competency in avoiding an excessive posterior slope change, lateral hinge fracture, and undercorrection with increased surgical experience. However, the surgeons was not able to achieve competency in prevention of overcorrection after 100 procedures. The incidence of surgical failures in MOWHTO has varied widely in previous studies ${ }^{7,814-1,21,22)}$, ranging from $9 \%$ to $62 \%$ for undercorrection, from $4 \%$ to $16 \%$ for overcorrection, and from $1.5 \%$ to $30.4 \%$ for lateral hinge fracture. These wide ranges may be due to different definitions of each surgical error and different surgical techniques. Our results suggest that the incidence of undercorrection, lateral hinge fracture, and excessive posterior slope change could be reduced by surgical experience in MOWHTO; therefore, the differences in the incidence of surgical errors in MOWHTO may be due to different learning curves of the surgeons. 

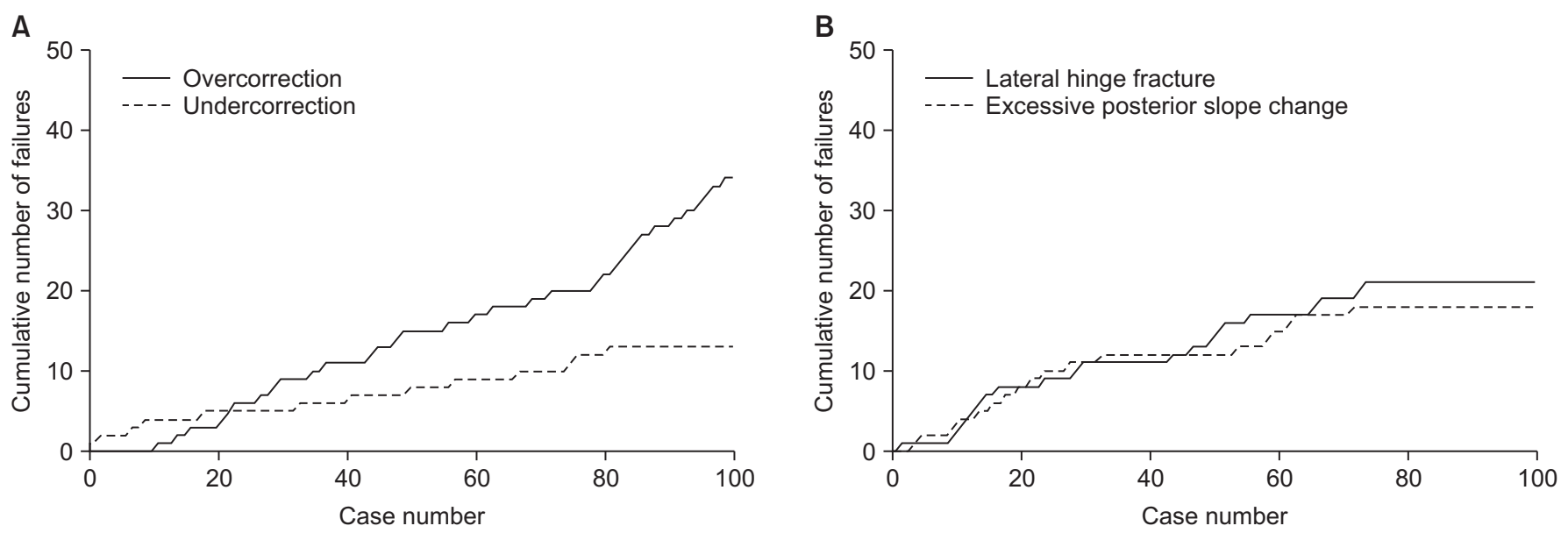

Fig. 1. Cumulative number of failures for each surgical factor. (A) Overcorrection shows an increasing tendency as surgical experience increases while undercorrection shows a decreasing tendency. (B) Excessive posterior slope change and lateral hinge fracture show a decreasing tendency.
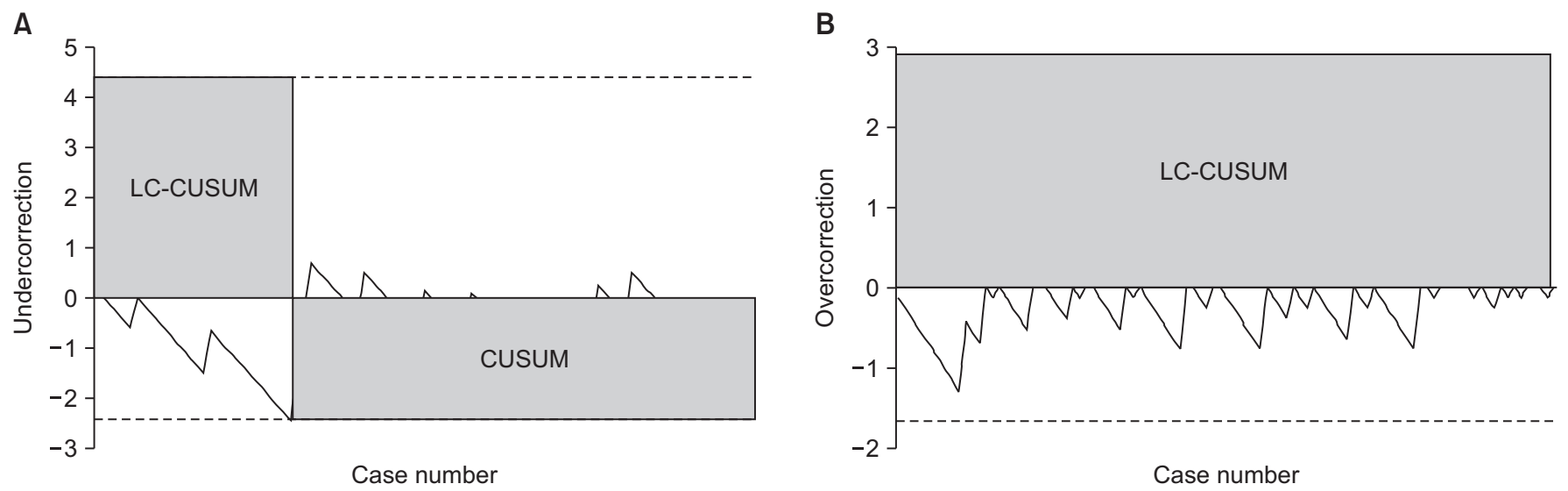

Fig. 2. Cumulative summation test for learning curve (LC-CUSUM) and standard cumulative summation (CUSUM) test for undercorrection and overcorrection. (A) The LC-CUSUM reached the lower decision limit after 27 procedures in preventing undercorrection, suggesting surgical competency could be achieved. (B) The LC-CUSUM did not reach the lower decision limit after 100 procedures in prevention of overcorrection, suggesting surgical competency could not be achieved.

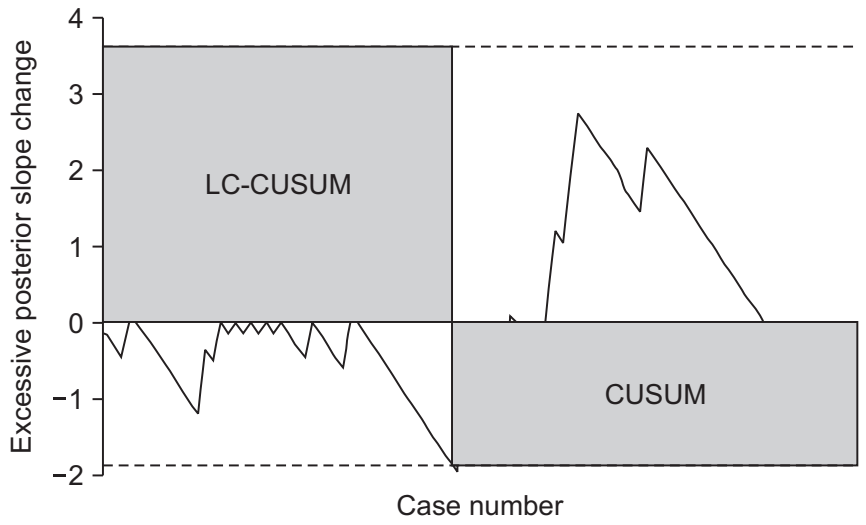

Fig. 3. Cumulative summation test for learning curve (LC-CUSUM) and standard cumulative summation (CUSUM) test for posterior slope. The LC-CUSUM reached the lower decision limit after 47 procedures in preventing excessive posterior slope change, suggesting surgical competency could be achieved.

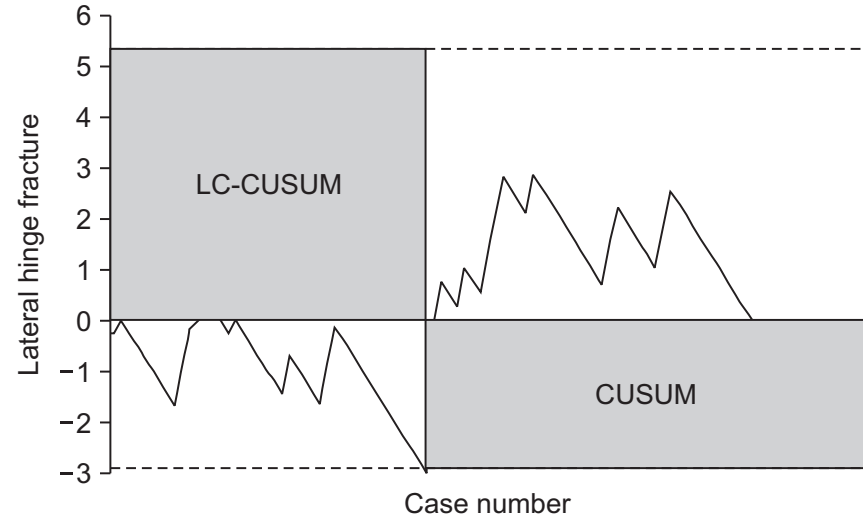

Fig. 4. Cumulative summation test for learning curve (LC-CUSUM) and standard cumulative summation (CUSUM) test for lateral hinge fracture. The LC-CUSUM reached the lower decision limit after 42 procedures in preventing lateral hinge fracture, suggesting surgical competency could be achieved. 
Our results showed that most of surgical errors could be improved as the surgical volume increases. By contrast, the incidence of overcorrection showed an increasing tendency as surgical experience increases. Several studies ${ }^{23-27)}$ have reported that overcorrection in MOWHTO is associated with soft tissue laxity. Kyung et al. ${ }^{24)}$ have reported that bony correction in MOWHTO could be consistently achieved in their navigation study; however, the bony correction was not consistent with the change of mechanical limb alignment. The authors also remarked that soft tissue laxity could affect the difference. Ogawa et al. ${ }^{28)}$ have also reported that an additional alignment change due to soft tissue laxity could occur in MOWHTO. However, conventional planning techniques primarily reflect varus deformity without considering soft tissue laxity, which may explain the increasing tendency in the rate of overcorrection as the surgical volume increases. Another possible explanation is that surgical experience diminished the incidence of undercorrection and lateral hinge fracture, both of which could affect the bony correction. As bony correction of the tibia becomes more accurate, the effect of additional alignment change due to soft tissue laxity became prominent. As other authors ${ }^{23-27)}$ have already remarked, soft tissue laxity could change the extent of correction additionally in MOWHTO. These results suggest that conventional techniques used to decide correction angle may be inappropriate for achieving a targeted limb alignment and preventing overcorrection. Thus, changing the target point to less than Fujisawa point (less than $62.5 \%$ of tibia plateau width), depending on the amount of medial soft tissue laxity of the knee joint, might be appropriate for preventing overcorrection as Ogawa et al. ${ }^{28)}$ already recommended.

Another interesting finding is that LC-CUSUM revealed improvement in surgical errors with time, despite no significant difference in the incidence of surgical errors between early and late periods. A possible explanation for the difference might be related to limitation of conventional incidence-based measurements. Conventional incidence-based measurements could not detect statistically small differences. However, the LC-CUSUM analysis could confirm that surgeons attained the predefined competency and detect surgical learning curves elaborately. That could be the reason for the difference, and suggests that incidence-based conventional measurements are a limited method for evaluating learning curves. Biau et al. ${ }^{5)}$ also reported that the LC-CUSUM analysis could be a useful method in evaluating the continuous quality improvement and learning curves.

There was some limitations in this study. Firstly, analysis of the learning curve for biplane MOWHTO was done only for a single surgeon. So our findings could not be representative of surgeons with other levels of expertise and could be biased depending upon the level of skill and talent of the surgeon. Additional studies including more surgeons with different levels of surgical experience or surgical volumes, are needed for generalization of our results. In spite of this limitation, our study could provide a significant message that some of the technical errors in MOWHTO might be improved by the surgical experience of a novice surgeon. Also the results indicate indirectly that surgical experience might not improve overcorrection in biplane MOWHTO with conventional planning techniques and surgeons should consider soft tissue laxity in determining the correction angle to achieve the targeted alignment. Secondly, the study analyzed the data retrospectively although the LC-CUSUM could provide feedback and reduce the potential of failure in a prospective study as well. However, there was a benefit of the retrospective design. The result was influenced only by surgical volume, without being affected by prospective feedback by the LC-CUSUM analysis. Thirdly, the study was evaluated with simple radiographs; therefore, observational bias and rotation of tibia or femur might have affected the value of measurements in overcorrection or undercorrection and posterior slope. Lastly, the operation time and rare complications, such as nonunion and neurovascular injury, could not be analyzed with the LC-CUSUM analysis.

\section{Conclusions}

Undercorrection, excessive posterior slope change, and lateral hinge fracture could be improved by surgical experience. However, it did not reduce the incidence of overcorrection in MOWHTO until 100 cases conducted over 6 years. These findings suggest that the conventional technique used to decide correction angle has a limitation in achieving a targeted alignment, and other preoperative planning methods with additional consideration into soft tissue laxity are needed to prevent unexpected overcorrection in MOWHTO.

\section{Conflict of Interest}

No potential conflict of interest relevant to this article was reported.

\section{Acknowledgments}

We appreciate Young-Kyun Lee for his effort in providing medical advice for LC-CUSUM analysis in this study. 


\section{References}

1. Black KP, Armstrong AD, Hutzler L, Egol KA. Quality and safety in orthopaedics: learning and teaching at the same time: AOA critical issues. J Bone Joint Surg Am. 2015;97: 1809-15.

2. Lee YK, Biau DJ, Yoon BH, Kim TY, Ha YC, Koo KH. Learning curve of acetabular cup positioning in total hip arthroplasty using a cumulative summation test for learning curve (LC-CUSUM). J Arthroplasty. 2014;29:586-9.

3. Zhang Q, Zhang Q, Guo W, Liu Z, Cheng L, Yue D, Zhang $\mathrm{N}$. The learning curve for minimally invasive Oxford phase 3 unicompartmental knee arthroplasty: cumulative summation test for learning curve (LC-CUSUM). J Orthop Surg Res. 2014;9:81.

4. Hodgins JL, Veillette C, Biau D, Sonnadara R. The knee arthroscopy learning curve: quantitative assessment of surgical skills. Arthroscopy. 2014;30:613-21.

5. Biau DJ, Williams SM, Schlup MM, Nizard RS, Porcher R. Quantitative and individualized assessment of the learning curve using LC-CUSUM. Br J Surg. 2008;95:925-9.

6. Lee YK, Ha YC, Hwang DS, Koo KH. Learning curve of basic hip arthroscopy technique: CUSUM analysis. Knee Surg Sports Traumatol Arthrosc. 2013;21:1940-4.

7. Hankemeier S, Mommsen P, Krettek C, Jagodzinski M, Brand J, Meyer C, Meller R. Accuracy of high tibial osteotomy: comparison between open- and closed-wedge technique. Knee Surg Sports Traumatol Arthrosc. 2010;18:132833.

8. Van den Bempt M, Van Genechten W, Claes T, Claes S. How accurately does high tibial osteotomy correct the mechanical axis of an arthritic varus knee? A systematic review. Knee. 2016;23:925-35.

9. Nha KW, Kim HJ, Ahn HS, Lee DH. Change in posterior tibial slope after open-wedge and closed-wedge high tibial osteotomy: a meta-analysis. Am J Sports Med. 2016;44:300613.

10. Sprenger TR, Doerzbacher JF. Tibial osteotomy for the treatment of varus gonarthrosis: survival and failure analysis to twenty-two years. J Bone Joint Surg Am. 2003;85:469-74.

11. Amis AA. Biomechanics of high tibial osteotomy. Knee Surg Sports Traumatol Arthrosc. 2013;21:197-205.

12. Miller BS, Downie B, McDonough EB, Wojtys EM. Complications after medial opening wedge high tibial osteotomy. Arthroscopy. 2009;25:639-46.

13. Kazimoglu C, Akdogan Y, Sener M, Kurtulmus A, Karapinar
$\mathrm{H}$, Uzun B. Which is the best fixation method for lateral cortex disruption in the medial open wedge high tibial osteotomy? A biomechanical study. Knee. 2008;15:305-8.

14. Dexel J, Fritzsche H, Beyer F, Harman MK, Lutzner J. Openwedge high tibial osteotomy: incidence of lateral cortex fractures and influence of fixation device on osteotomy healing. Knee Surg Sports Traumatol Arthrosc. 2017;25:832-7.

15. Giffin JR, Vogrin TM, Zantop T, Woo SL, Harner CD. Effects of increasing tibial slope on the biomechanics of the knee. Am J Sports Med. 2004;32:376-82.

16. Iorio R, Pagnottelli M, Vadalà A, Giannetti S, Di Sette P, Papandrea P, Conteduca F, Ferretti A. Open-wedge high tibial osteotomy: comparison between manual and computerassisted techniques. Knee Surg Sports Traumatol Arthrosc. 2013;21:113-9.

17. Brosset T, Pasquier G, Migaud H, Gougeon F. Opening wedge high tibial osteotomy performed without filling the defect but with locking plate fixation ( $\mathrm{TomoFix}^{\mathrm{TM}}$ ) and early weight-bearing: prospective evaluation of bone union, precision and maintenance of correction in 51 cases. Orthop Traumatol Surg Res. 2011;97:705-11.

18. Lee DH, Han SB, Oh KJ, Lee JS, Kwon JH, Kim JI, Patnaik S, Shetty GM, Nha KW. The weight-bearing scanogram technique provides better coronal limb alignment than the navigation technique in open high tibial osteotomy. Knee. 2014; 21:451-5.

19. El-Azab HM, Morgenstern M, Ahrens P, Schuster T, Imhoff AB, Lorenz SG. Limb alignment after open-wedge high tibial osteotomy and its effect on the clinical outcome. Orthopedics. 2011;34:e622-8.

20. Biau DJ, Resche-Rigon M, Godiris-Petit G, Nizard RS, Porcher R. Quality control of surgical and interventional procedures: a review of the CUSUM. Qual Saf Health Care. 2007;16:203-7.

21. Stanley JC, Robinson KG, Devitt BM, Richmond AK, Webster KE, Whitehead TS, Feller JA. Computer assisted alignment of opening wedge high tibial osteotomy provides limited improvement of radiographic outcomes compared to flouroscopic alignment. Knee. 2016;23:289-94.

22. Takeuchi R, Ishikawa H, Kumagai K, Yamaguchi Y, Chiba N, Akamatsu Y, Saito T. Fractures around the lateral cortical hinge after a medial opening-wedge high tibial osteotomy: a new classification of lateral hinge fracture. Arthroscopy. 2012;28:85-94.

23. Marti CB, Gautier E, Wachtl SW, Jakob RP. Accuracy of frontal and sagittal plane correction in open-wedge high 
tibial osteotomy. Arthroscopy. 2004;20:366-72.

24. Kyung BS, Kim JG, Jang KM, Chang M, Moon YW, Ahn JH, Wang JH. Are navigation systems accurate enough to predict the correction angle during high tibial osteotomy? Comparison of navigation systems with 3-dimensional computed tomography and standing radiographs. Am J Sports Med. 2013;41:2368-74.

25. Sabharwal S, Zhao C. Assessment of lower limb alignment: supine fluoroscopy compared with a standing full-length radiograph. J Bone Joint Surg Am. 2008;90:43-51.

26. Sim JA, Kwak JH, Yang SH, Choi ES, Lee BK. Effect of weight-bearing on the alignment after open wedge high tibial osteotomy. Knee Surg Sports Traumatol Arthrosc. 2010; 18:874-8.

27. Heijens E, Kornherr P, Meister C. The coronal hypomochlion: a tipping point of clinical relevance when planning valgus producing high tibial osteotomies. Bone Joint J. 2016;98: 628-33.

28. Ogawa H, Matsumoto K, Ogawa T, Takeuchi K, Akiyama H. Preoperative varus laxity correlates with overcorrection in medial opening wedge high tibial osteotomy. Arch Orthop Trauma Surg. 2016;136:1337-42. 